EDUR • Educação em Revista. 2020; 36: e216356 DOI: http://dx.doi.org/10.1590/0102-4698216356

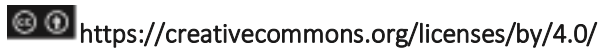

\title{
ARTíCULO
}

\section{FACTORES DETERMINANTES DEL USO DE DISPOSITIVOS MÓVILES EN LA FORMACIÓN INICIAL DOCENTE DE URUGUAY}

\author{
CARLOS MARCELO ${ }^{1}$ \\ ORCID: https://orcid.org/0000-0002-8547-367X \\ CARMEN YOT ${ }^{2}$ \\ ORCID: https://orcid.org/0000-0001-9447-8993 \\ EDUARDO RODRIGUEZ ZIDAN ${ }^{3}$ \\ ORCID: https://orcid.org/0000-0002-6437-578X \\ JUAN PABLO ZORRILLA-SALGADOR ${ }^{4}$ \\ ORCID: https://orcid.org/0000-0001-9786-6457
}

\begin{abstract}
RESÚMEN: Actualmente en América Latina y el Caribe se han implementado políticas para promover la integración de las TIC en los centros educativos, aumentando la inversión públicoprivada en programas OLPC. Sin embargo, todavía es insuficiente el conocimiento sobre cómo se están integrando las tecnologías digitales en la enseñanza. El objetivo principal de este artículo es analizar el uso que del ordenador portátil hacen los profesores en Uruguay en formación. El diseño de esta investigación es de tipo mixto de dominancia cuantitativa. Se desarrolló un análisis descriptivo correlacional no experimental transaccional mediante encuesta a estudiantes de profesorado que se complementó con entrevistas semiestructuradas. Se encontró que los estudiantes usan poco la tecnología proporcionada por el Estado como recurso para el aprendizaje y entre aquellos que si la usan, es mayor el uso del ordenador portátil en las asignaturas curriculares de la especialidad de profesorado frente a las asignaturas pedagógicas. No se encontró evidencia de que el género, la edad, la especialidad y el nivel cursado, sean características que influyan sobre la percepción de utilidad que tienen los estudiantes de los ordenadores portátiles.
\end{abstract}

Palabras clave: formación inicial docente, tecnología digital, dispositivos móviles, diseño mixto, modelo logit.

\footnotetext{
${ }^{1}$ Universidad de Sevilla. Sevilha, Espanha. <marcelo@us.es>

${ }^{2}$ Universidad de Sevilla. Sevilha, Espanha. <carmenyot@us.es>

${ }^{3}$ Universidad ORT Uruguay. Montevidéu, Uruguai. <rodriguez_ce@ort.edu.uy>

${ }^{4}$ Universidad ORT Uruguay. Montevidéu, Uruguai. <juanpablozorrilla@gmail.com> Educação em Revista | Belo Horizonte|v.36|e216356|2020
} 


\title{
FATORES DETERMINANTES NO USO DE DISPOSITIVOS MÓVEIS NA FORMAÇÃO INICIAL DOCENTE NO URUGUAI
}

RESUMO: Atualmente, na América Latina e no Caribe, foram implementadas políticas para promover a integração das TICs nos sistemas escolares, aumentando o investimento em programas OLPC. Entretanto, ainda não há conhecimento suficiente sobre como essas tecnologias digitais estão sendo integradas no esino. O objetivo principal deste artigo é analisar o uso que os professores em formação, no Uruguai, fazem do computador portátil. O desenho desta pesquisa é de tipo misto, de dominância quantitativa. Foi desenvolvida uma análise descritivo-correalcional não experimental transacional a partir de questionários envolvendo estudantes de licenciatura, complementados com entrevistas semi-estruturadas. Identificamos que os estudantes utilizam pouco a tecnología proporcionada pelo Estado como recurso para a aprendizagem, e entre aqueles que os utilizam, é maior o uso do computador portátil para as disciplinas específicas dos cursos do que para as disciplinas pedagógicas. Não foram encontradas evidências de que o gênero, a idade, a especialidade e o nível cursado sejam características que influam sobre a percepção de utilidade que os estudantes têm dos computadores portáteis.

Palavras chave: Formação inicial docente, tecnología digital, dispositivos móveis, desenho misto, modelo logit.

\section{FACTORS DETERMINANTS USE OF MOBILE DEVICES IN THE INITIAL TEACHER TRAINING IN URUGUAY}

\begin{abstract}
In Latin America and the Caribbean, policies have been implemented to promote the integration of ICTs in schools, increasing public-private investment in OLPC programs. However, there is still insufficient knowledge about how digital technologies are being integrated into education. The main objective of this article is to analyze the use of the laptop by teachers in training in Uruguay. The design of this research is of a mixed type of quantitative dominance. A descriptive correlational non-transactional experimental analysis was developed through a survey of teacher students that was complemented with semi-structured interviews. It was found that students use little of the technology provided by the State as a resource for learning, and among those who do use it, the use of laptop computers in curricular subjects of the teaching specialty is greater than in pedagogical subjects. No evidence was found that gender, age, specialty, and level are characteristics that influence students' perception of the usefulness of laptops.
\end{abstract}

Keywords: initial teacher training, digital technology, mobile devices, mixed design, logit model. 


\section{INTRODUCCIÓN}

En el contexto de América Latina y Caribe, Uruguay es uno de los países de la región con mayor nivel de cobertura de Internet y desarrollo de banda ancha con niveles de costo similares a los países de la OCDE (Galpering, 2017) y, junto a Chile y Brasil, con mayor penetración de tecnología de la información y comunicación (en adelante, TIC) en hogares y centros educativos (Cabrera Borges, et al., 2018; Sunkel, Trucco y Espejo 2014; Jara, 2015).

La política TIC en Uruguay, pionera en la región, favorece un escenario de expansión tecnológica único. En el año 2007 se creó un programa nacional de acceso universal a Internet y a recursos tecnológicos gratuitos, como computadores portátiles (laptops), cuyo propósito fundamental era la promoción de la equidad social, la inclusión digital y la mejora de la calidad educativa. El Plan CEIBAL continua con la entrega gratuita (y reposición o servicio técnico necesario) de laptops a la totalidad de los estudiantes de educación básica, a todos los docentes de educación primaria y a los estudiantes de profesorado cuando estos comienzan a cursar el tercer año de carrera. El propósito de este estudio es, precisamente, aportar conocimiento sobre las experiencias de uso del laptop para el aprendizaje en las carreras de pedagogía según son percibidas por los propios estudiantes, en un contexto internacional de surgimiento de nuevas pedagogías para el aprendizaje que se apoyan en las tecnologías digitales (Chaia et al., 2017; Ferguson y Buckingham, 2012; Fullan, 2013).

\section{Notas conceptuales}

Las tecnologías digitales se han configurado como exponente de innovación educativa. Así, la mayoría de los gobiernos de los diferentes países han implementado políticas para promover la integración de las TIC en los centros educativos y aulas. En algunos países se ha dotado a los alumnos de computadores en un modelo 1 a 1 . En otros se han proporcionado a las escuelas dispositivos electrónicos y se ha hecho un especial énfasis en la formación de los docentes. Sin embargo, a pesar de la inversión en equipamiento y formación, los informes nos muestran serias dificultades para la integración de las tecnologías en los procesos de enseñanza- aprendizaje y en la gestión escolar (Fernández, Cebreiro y Fernández, 2014; Hammond, 2014; Hinostroza et al., 2011).

La necesidad de repensar la formación del profesorado, en especial la formación inicial docente, aparece entonces como una de las prioridades a abordar (Brecko, Kampylis y Punie, 2014). Los profesores en formación necesitan ser formados con tecnologías digitales (Gutiérrez, Palacios y Torrego, 2010). Se requiere el esfuerzo por parte de sus formadores de usar las TIC pedagógicamente, y de manera sostenida, porque impacta positivamente en las actitudes de los futuros docentes (Messina y Tabone, 2013), las cuales son el factor más influyente en la intención de uso posterior (Yusop, 2015). También deben llegar a ser capaces de aprender con dichas tecnologías. El aprendizaje autorregulado con tecnologías digitales entre los estudiantes universitarios es débil (Rodríguez-Zidan, et al., 2017). Requieren, como plantean Lai, Wang y Lei (2012), del apoyo, consejo y motivación por parte de sus docentes.

A este respecto, la realidad no se dibuja como nos gustaría. Aun cuando los docentes en formación, así como sus formadores, se muestran a favor de utilizar las TIC en actividades de aprendizaje dentro y fuera del aula, lo cierto es que en los programas se dan escasas oportunidades instruccionales (Gülbahar, 2008). Si bien las tecnologías digitales, especialmente las herramientas 2.0, facilitarían y enriquecerían el desarrollo de los procesos ligados al aprendizaje profesional docente, observamos que no han transformado la forma como se diseña el aprendizaje de los futuros docentes (Mcloughlin, 2013). El estado de la cuestión se refleja muy bien cuando se identifican diferentes perfiles de formadores frente a la innovación pedagógica en la enseñanza con TIC (Avidov-Ungar y Iluz, 2014; Kopcha, Rieber y Walker, 2016). Y en este sentido, los resultados de las investigaciones previas muestran una clara debilidad de los formadores en el uso de las tecnologías. Una reciente revisión de estudios sobre el conocimiento tecnopedagógico de 
estos (Uerz, Volman y Kral, 2018) muestra la débil competencia de los formadores. Lo que plantea la necesidad de profundizar en las competencias tecnológicas, en las competencias pedagógicas relacionadas con las tecnologías, en las creencias acerca de la enseñanza y el aprendizaje con tecnologías y en las competencias profesionales hacia la innovación docente.

Para que los docentes en formación tengan oportunidades de integrar las tecnologías digitales, se requiere no sólo garantizar que cada uno de ellos disfrute de acceso a las mismas sino también que sus formadores posean competencias digitales (Aslan y Zhu 2015; Kalonde y Mousa, 2016). En el contexto internacional se están llevando a cabo iniciativas y estrategias para facilitar la adopción de las tecnologías digitales en la formación docente y así lograr la demandada transferencia (Bakir, 2016). En Uruguay, el Plan CEIBAL ha impulsado la innovación tecnológica en los centros educativos con la dotación de ordenadores a estudiantes y docentes y acceso a Internet (Pittaluga y Rivoir, 2012; Rodríguez Zidan et al., 2017) y ha puesto de manifiesto la necesidad de atender a la formación inicial docente al considerar a esta población como beneficiaria.

Proveer a los profesores en formación de un ordenador portátil para uso personal es una excelente oportunidad de ampliar las experiencias de aprendizaje hacia el aprendizaje móvil (Baran, 2014). La previsión de la UNESCO (2013) es que en los próximos quince años el aprendizaje móvil se integre más en el sistema educativo ordinario. ¿Y esto qué supone? Hace relativamente poco tiempo nos bastaba con que el aprendizaje tuviera lugar apoyado en dispositivos móviles para hablar de aprendizaje móvil (Dyson et al., 2009). Sin embargo, en la actualidad, se asume que el uso de los dispositivos móviles en el aula ha de plantearse como parte de un proceso de transformación más amplio de la enseñanza (Ramírez-Montoya y García-Peñalvo, 2017).

De acuerdo con el estudio desarrollado por Sad y Gokta (2014), los docentes en formación perciben el laptop como un dispositivo más adecuado que el teléfono móvil para el aprendizaje y valoran muy positivamente de él, entre otros aspectos, el que les permita disfrutar de oportunidades de aprendizaje individualizado. Sin embargo, su potencialidad está en muchos casos infravalorada. Hughes (2013) evidenció como en un programa de formación basado en el uso de la laptop, los docentes en formación estaban expuestos a las mismas actividades a las que hubiesen estado una década atrás. Cabía esperar que el uso del laptop modificara los procesos formativos pero los resultados no avalaron esta expectativa (Hughes, Liu y Lim, 2016).

El uso de dispositivos electrónicos, como el ordenador portátil, permite a los estudiantes ser más independientes en su esfuerzo de aprender, lo que refuerza sus habilidades de aprendizaje a lo largo de la vida (Newhouse, Williams y Pearson, 2006). Por otra, al incorporarlo a un programa de formación docente se contribuye a que los participantes ganen en autoconfianza e intención de uso en el futuro (Kay y Knaack, 2005). Además, los docentes que tienen un laptop a su disposición pasan a manifestar una actitud más positiva hacia las tecnologías digitales que los que no cuentan con él (Sipilä, 2010). Donovan, Green y Hansen (2011) corroboran que los docentes en formación no sólo aumentan el nivel de habilidad con la tecnología sino también las creencias sobre los usos educativos de las TIC, pero para ello se precisa un uso del portátil permanente y extensible a todo el programa.

¿Qué diferencias pueden existir entre usar dispositivos móviles o no? Un estudio desarrollado por Sung, Chang y Liu (2016) ha revelado que el efecto sobre el aprendizaje de los estudiantes del uso de dispositivos móviles es mayor que si no se utilizan o si sólo se emplean ordenadores de sobremesa. Pero para que los efectos sean perceptibles se necesita que los formadores los integren en el diseño y desarrollo de la formación que imparten de manera significativa. En este sentido, percibir el dispositivo móvil como un instrumento apto para facilitar el andamiaje del aprendizaje, la colaboración y la creación de contenidos guiaría con éxito el proceso de enseñanza (Herro, Kiger y Owens, 2013). Y es que los dispositivos móviles se ajustan al aprendizaje docente al proporcionar oportunidades nunca antes accesibles para la observación, la producción de conocimiento, la reflexión, la retroalimentación, etc. (Aubusson, Schuck y Burden, 2009). 


\section{METODOLOGÍA}

En base a la revisión de investigaciones anteriormente descritas, nos planteamos en este estudio los siguientes objetivos: describir el uso que del ordenador portátil hacen los docentes en formación dentro y fuera del contexto formal de aula, analizar la percepción acerca de cómo el laptop condiciona la forma de aprender y determinar la incidencia sobre el uso manifestado y la valoración del laptop de una serie de variables independientes seleccionadas como el género, la edad, la modalidad de cursado y la especialidad de la carrera de pedagogía. Se profundizará sobre las representaciones de los estudiantes acerca de la utilidad del laptop en su proceso de formación inicial, al tiempo que se pretende explorar las autopercepciones de los estudiantes acerca de los factores explicativos del empleo que hacen del laptop en la formación docente.

Para ello se ha recurrido a un método mixto de investigación educativa, el cual nos permitía articular y combinar datos de naturaleza cuantitativa y cualitativa a efectos de enriquecer la recogida y análisis de datos desde múltiples perspectivas. En particular, el diseño de esta investigación se corresponde con el modelo incrustado de dominancia cuantitativa (Creswell y Plano, 2007). En este sentido, en una primera fase, el abordaje cuantitativo privilegió el análisis descriptivo correlacional no experimental transaccional, tipo "survey". Se adaptó y validó la encuesta "Survey of Mobile device use during" (Witecki y Nonnencke, 2015). Por medio de ella, el nivel de uso y las percepciones de los estudiantes sobre la utilidad del laptop pudieron ser analizados. Luego se desarrollaron diferentes modelos de contraste de hipótesis mediante modelos de regresión. En la segunda etapa se buscó revelar las percepciones de los sujetos desde un enfoque cualitativo (Flick, 2015). El estudio cualitativo se basó en la aplicación de entrevistas semiestructuradas a estudiantes de los cursos de didáctica de las modalidades de cursado presencial y no presencial.

\section{Participantes}

Para el abordaje cuantitativo del estudio se siguió un muestreo no probabilístico accidental. Hubo un primer criterio de selección, a saber: la tenencia de un ordenador portátil entregado gratuitamente a cada estudiante en el marco del Plan CEIBAL. Los datos facilitados por las autoridades educativas nos permitió identificar una población inicial de 2250 estudiantes de profesorado, los cuales reunían este criterio. La muestra finalmente quedó constituida por aquellos estudiantes que accedieron a responder al instrumento cuantitativo. Esto es, un total de 385 estudiantes de profesorado. Como se observa en la Tabla 1 , de ellos, el $73 \%$ son mujeres y el $27 \%$ hombres. El promedio de edad es de 35 años. El 47\% estudia carreras de ciencias experimentales, de ciencias de la naturaleza o exactas. Con relación a la modalidad de estudio, el 67.3\% está inscrito en la modalidad presencial mientras el resto en un modelo hibrido de formación, con un alto componente de educación virtual.

Tabla 1. Análisis de frecuencias de la muestra de estudio.

\begin{tabular}{lcc}
\hline \multicolumn{1}{c}{ Variable } & Frecuencia & Porcentaje \\
\hline Rango de edad & & \\
Edad 18 a 30 & 130 & $33.9 \%$ \\
31 a 40 & 137 & $35.4 \%$ \\
41 a 50 & 97 & $25.1 \%$ \\
51 y mas & 22 & $5.7 \%$ \\
Edad 18 a 30 & 130 & $33.9 \%$ \\
Género & & \\
Femenino & 281 & $73 \%$ \\
Masculino & 104 & $27 \%$ \\
Modalidad & & \\
No presencial & 126 & $32.7 \%$
\end{tabular}

Educação em Revista | Belo Horizonte|v.36|e216356|2020 
Presencial

\section{Especialidad}

Humanidades

Ciencias

Número de observaciones
$67.3 \%$

$52.5 \%$

$44.2 \%$

Fuente: elaboración propia.

Con relación a la muestra de participantes para el enfoque cualitativo del estudio, se aplicó un muestreo intencional. Las entrevistas semiestructuradas se realizaron a 8 estudiantes. Su selección tuvo lugar atendiendo a que representaran combinaciones diferentes de dos estratos de interés para la investigación: modalidad de cursado y especialidad de la carrera de profesorado.

\section{Instrumento y técnica}

La adaptación de la encuesta "Survey of Mobile device use during" (Witecki y Nonnencke, 2015) resultó en un instrumento constituido por 45 reactivos, como se observa en la Tabla 2. Éste incluyó un primer grupo de preguntas de carácter demográficas que permitían abordar el perfil del estudiantado y el nivel de acceso de estos a las tecnologías. Seguidamente mediante escala Likert se cuestionaba acerca del uso del laptop que hacen en su formación docente, pero en tres tipos de escenarios: centro educativo y de práctica pre profesional y en el hogar. Por último, un total de siete preguntas abiertas pretendían indagar sobre la autopercepción de los futuros docentes sobre sus experiencias de uso pedagógico del laptop.

Tabla 2. Dimensiones, variables y tipos de preguntas del instrumento aplicado.

\begin{tabular}{|c|c|c|c|}
\hline Dimensión & Descripción de variables & Ítems & $\begin{array}{c}\text { Tipo de } \\
\text { preguntas }\end{array}$ \\
\hline Sociodemográfica & Género y Edad. & $1-2$ y 6 & $\begin{array}{l}\text { Politómicas y } \\
\text { cuantitativas }\end{array}$ \\
\hline Académica & $\begin{array}{l}\text { Año ingreso, Nivel que cursa, } \\
\text { Especialidad y Modalidad de } \\
\text { cursado. }\end{array}$ & $3-5$ y $7-8$ & $\begin{array}{l}\text { Dicotómicas, } \\
\text { politómicas y } \\
\text { respuesta } \\
\text { múltiple }\end{array}$ \\
\hline Acceso a TIC & $\begin{array}{l}\text { Acceso a diferentes tipos de recursos } \\
\text { digitales, Acceso a Internet, Tipo de } \\
\text { Conexión y Soporte. }\end{array}$ & $9-17$ & Escala Likert \\
\hline $\begin{array}{ll}\text { Uso } & \text { de } \\
\text { computadoras } \\
\text { portátiles en el } \\
\text { centro educativo y } \\
\text { centro de práctica }\end{array}$ & $\begin{array}{l}\text { Acceso y frecuencia de uso de } \\
\text { computadoras portátiles en el centro } \\
\text { del formación inicial y en la práctica } \\
\text { pre profesional*. }\end{array}$ & $\begin{array}{c}18-26 \text { y } \\
29\end{array}$ & Escala Likert \\
\hline $\begin{array}{l}\text { Uso de } \\
\text { computadoras } \\
\text { portátiles fuera del } \\
\text { centro educativo }\end{array}$ & $\begin{array}{l}\text { Acceso y frecuencia de uso de } \\
\text { computadoras portátiles fuera del } \\
\text { formación inicial y en la práctica pre } \\
\text { profesional**. }\end{array}$ & $\begin{array}{c}30-37 \text { y } \\
40-42\end{array}$ & Escala Likert \\
\hline $\begin{array}{l}\text { Autopercepción del } \\
\text { cambio educativo a } \\
\text { partir del uso de } \\
\text { portátiles }\end{array}$ & $\begin{array}{l}\text { Autopercepción de cambio } \\
\text { educativo basado en dispositivos } \\
\text { móviles y relato de buenas prácticas } \\
\text { de uso pedagógico de dispositivos } \\
\text { móviles. }\end{array}$ & $\begin{array}{l}27-28 y \\
38-39 y \\
43-45\end{array}$ & $\begin{array}{l}\text { Preguntas } \\
\text { abiertas }\end{array}$ \\
\hline
\end{tabular}


* Items: Navegar por Internet para el seguimiento de las clases, Usar redes sociales, Usar software específico de la asignatura, Crear presentaciones, documentos, videos, Entrar a la plataforma CREA2, responder a tareas de evaluación, uso del dispositivo en la práctica profesional

** Items: Navegar en Internet para hacer tareas de estudio, navegación con fines de entretenimiento, usar el correo electrónico, usar redes sociales, bajar, subir y buscar material del sitio web de tu Centro educativo, hacer tareas domiciliarias elaborar presentaciones multimedia, participar en comunidades virtuales entrar a una plataforma educativa, producir materiales, usar software específico de mi asignatura, publicar y mantener un sitio web personal.

Fuente: elaboración propia.

Los resultados acerca del grado de fiabilidad y consistencia interna de las escalas utilizadas se presentan en la Tabla 3.

Tabla 3. Niveles de confiabilidad de las escalas de medición utilizadas.

\begin{tabular}{|l|c|c|c|}
\hline \multicolumn{1}{|c|}{ Escalas } & N & $\begin{array}{c}\text { Alpha de } \\
\text { Cronbach }\end{array}$ & Decisión(*) \\
\hline $\begin{array}{l}\text { Escala para medir acceso y frecuencia de uso } \\
\text { del ordenador portátil en las aulas del centro } \\
\text { de formación inicial y en la práctica pre } \\
\text { profesional }\end{array}$ & 299 & 0.863 & Muy buena \\
\hline $\begin{array}{l}\text { Escala para medir acceso y frecuencia de uso } \\
\text { del ordenador portátil fuera del centro de } \\
\text { formación inicial }\end{array}$ & 292 & 0.929 & Excelente \\
\hline
\end{tabular}

* Se aplica el criterio interpretativo sugerido por Jisu, DeLorme y Reid (2006), quienes recomiendan que para estudios confirmatorios el valor debe ser superior al valor de 0.80 .

Fuente: elaboración propia.

Los datos expresan que en las tres escalas consideradas, los ítems están fuertemente correlacionados. Los valores observados dan cuenta de la consistencia interna y, en consecuencia, miden un mismo constructo teórico.

El instrumento resultante tras la traducción de la encuesta primitiva y sucesivas rondas de ajustes para responder al contexto nacional por los integrantes del equipo de investigación fue validado por expertos. Tras ello, fue desarrollado en la plataforma LimeSurvey y enviado a los 2250 mails personales de los estudiantes de manera gradual durante los meses de julio a setiembre de 2017. En su envío se cuidó su presentación y se aseguró el anonimato a efectos de aumentar la confianza de los estudiantes.

Por su parte, la entrevista fue semiestructurada. Se contó, por ello, con un guion que fue validado por expertos en didáctica e investigadores en tecnologías educativas. Los encuentros para llevar a cabo la tarea de campo se realizaron en el propio centro de enseñanza, pero fuera del aula de clases. La duración promedio de cada entrevista fue de 40 minutos. El guion de entrevista se presenta en la Tabla 4.

Tabla 4. El guion de entrevistas.

\begin{tabular}{|l|l|}
\hline \multicolumn{1}{|c|}{ Dimensiones } & \multicolumn{1}{c|}{ Pregunta } \\
\hline $\begin{array}{l}\text { Valoración global acerca del impacto de las } \\
\text { tecnologías móviles }\end{array}$ & $\begin{array}{l}\text { ¿Qué opinión tiene acerca del impacto de } \\
\text { las tecnologías móviles en la formación de } \\
\text { profesores? ¿y del uso particular del } \\
\text { Ordenador portátil? }\end{array}$ \\
\hline Percepción de utilidad del dispositivo & $\begin{array}{l}\text { ¿Cómo valora la utilidad del uso del } \\
\text { ordenador portátil en las aulas de: }\end{array}$ \\
& $\begin{array}{l}\text { - Asignaturas específicas de su carrera } \\
\text { - Asignaturas del campo de las ciencias de la } \\
\text { educación } \\
\text { - En la práctica pre profesional }\end{array}$ \\
\hline
\end{tabular}

Educação em Revista | Belo Horizonte|v.36|e216356|2020 


\begin{tabular}{|l|l|}
\hline Experiencias exitosas & $\begin{array}{l}\text { ¿Podría relatar una experiencia exitosa del } \\
\text { uso del ordenador portátil como dispositivo } \\
\text { móvil que mejora la enseñanza y el } \\
\text { aprendizaje? }\end{array}$ \\
\hline $\begin{array}{l}\text { Percepción de impacto del uso del } \\
\text { ordenador portátil }\end{array}$ & $\begin{array}{l}\text { ¿Qué factores cree que pueden actuar como } \\
\text { facilitadores de la incorporación los } \\
\text { ordenadores portátiles en las aulas de } \\
\text { formación inicial? ey en las aulas de } \\
\text { educación media donde Usted realiza la } \\
\text { práctica docente pre profesional? }\end{array}$ \\
$\begin{array}{l}\text { ¿Qué factores cree que pueden actuar como } \\
\text { obstáculos o limitantes de la incorporación } \\
\text { de los ordenadores portátiles en las aulas de } \\
\text { formación inicial? ¿y en las aulas de } \\
\text { educación media donde usted realiza la } \\
\text { práctica docente pre profesional? }\end{array}$ \\
\hline
\end{tabular}

Fuente: elaboración propia.

\section{E1 proceso de análisis}

El proceso de análisis de los datos fue estructurado en base a los propósitos del estudio. El análisis de los datos recabados con la encuesta se realizó utilizando el software SPSS. En primer lugar, se recurrió a la estadística descriptiva (Media, Desviación Típica, Máximo, Mínimo, Varianza). Posteriormente, se utilizó un modelo de regresión logística (Modelo Logit) o también denominado modelo de respuesta cualitativa (Liu, Wilson y Paek, 2008; Peng y So, 2002). El objetivo de este procedimiento fue identificar las variables determinantes del uso de los ordenadores portátiles. Con la finalidad de adecuar el nivel de medición de las variables a las exigencias del Modelo Logit se adoptaron ciertas decisiones metodológicas. La Tabla 5 resume este proceso, indicando las decisiones asumidas, las categorías de las variables originales y las transformaciones realizadas.

Tabla 5. Proceso de transformación de las variables incluidas en los modelos Logit.

\begin{tabular}{|c|c|c|c|c|c|}
\hline \multirow[b]{2}{*}{ Variable } & \multirow{2}{*}{$\begin{array}{l}\text { Nivel de } \\
\text { medición }\end{array}$} & \multirow[b]{2}{*}{ Categorías } & \multicolumn{2}{|c|}{ Transformación } & \multirow{2}{*}{$\begin{array}{l}\text { Identificación } \\
\text { de categorías } \\
\text { del modelo }\end{array}$} \\
\hline & & & $\mathrm{Si}$ & No & \\
\hline Genero & Dicotómica & Masculino/Femenino & & $\mathrm{X}$ & $\begin{array}{l}\text { Femenino }=0 \\
\text { Masculino }=1\end{array}$ \\
\hline Edad & Ordinal & De 20 a 65 años & $\mathrm{X}$ & & $\begin{array}{l}35 \text { o menos }=0 \\
36 \text { o más }=1\end{array}$ \\
\hline Modalidad* & Politómica & $\begin{array}{l}\text { Presencial } \\
\text { IPA semipresencial } \\
\text { IFD semipresencial } \\
\text { CERP presencial } \\
\text { INET semipresencial }\end{array}$ & $\mathrm{X}$ & & $\begin{array}{l}\text { No presencial } \\
=0 \\
\text { Presencial }=1\end{array}$ \\
\hline $\begin{array}{l}\text { Carrera de } \\
\text { profesorado }\end{array}$ & Politómica & $\begin{array}{l}\text { Matemática, idioma } \\
\text { español, etc.** }\end{array}$ & $\mathrm{X}$ & & $\begin{array}{l}\text { Carrera de } \\
\text { Humanidades y } \\
\text { afines }=0 \\
\text { Carrera de } \\
\text { Ciencias } \\
\text { Naturales, }\end{array}$ \\
\hline
\end{tabular}

Educação em Revista | Belo Horizonte|v.36|e216356|2020 


\begin{tabular}{|c|c|c|c|c|c|}
\hline & & & & & $\begin{array}{l}\text { Matemática y } \\
\text { afines = } 1\end{array}$ \\
\hline $\begin{array}{l}\text { Nivel que } \\
\text { cursa }\end{array}$ & Politómica & $\begin{array}{l}\text { Tercer año } \\
\text { Cuarto año } \\
\text { Ya egreso }\end{array}$ & $\mathrm{X}$ & & $\begin{array}{l}\text { Ya egresó }=0 \\
\text { Tercer y cuarto } \\
\text { año }=1\end{array}$ \\
\hline $\begin{array}{l}\text { ¿Considera } \\
\text { que ha } \\
\text { cambiado su } \\
\text { forma de } \\
\text { aprender a } \\
\text { partir del } \\
\text { uso de la } \\
\text { CP? }\end{array}$ & Dicotómica & $\mathrm{Si} / \mathrm{No}$ & & $\mathrm{X}$ & $\begin{array}{l}\mathrm{No}=0 \\
\mathrm{Si}=1\end{array}$ \\
\hline $\begin{array}{l}\text { ¿Considera } \\
\text { que ha } \\
\text { cambiado su } \\
\text { forma de } \\
\text { aprender a } \\
\text { partir del } \\
\text { uso de } \\
\text { tecnologías? }\end{array}$ & Dicotómica & $\mathrm{Si} / \mathrm{No}$ & & $\mathrm{X}$ & $\begin{array}{l}\mathrm{No}=0 \\
\mathrm{Si}=1\end{array}$ \\
\hline $\begin{array}{l}\text { ¿Qué tan útil } \\
\text { le resulta la } \\
\text { CP en sus } \\
\text { clases de } \\
\text { profesorado? }\end{array}$ & $\begin{array}{l}\text { Ordinal } \\
\text { (Likert) }\end{array}$ & $\begin{array}{l}\text { Muy útil / Bastante } \\
\text { Útil / Poco útil / } \\
\text { Nada útil }\end{array}$ & $\mathrm{X}$ & & $\begin{array}{l}\text { Nada o poco } \\
\text { útil = } 0 \\
\text { Bastante o muy } \\
\text { útil = } 1\end{array}$ \\
\hline $\begin{array}{l}\text { Con el } \\
\text { apoyo de las } \\
\text { CP: ¿ } \mathrm{Ha} \\
\text { utilizado } \\
\text { aplicaciones } \\
\text { educativas } \\
\text { como apoyo } \\
\text { al } \\
\text { aprendizaje? }\end{array}$ & Dicotómica & $\mathrm{Si} / \mathrm{No}$ & & $\mathrm{X}$ & $\begin{array}{l}\mathrm{No}=0 \\
\mathrm{Si}=1\end{array}$ \\
\hline
\end{tabular}

* Se especifica la modalidad según los tipos de centro de formación docente en Uruguay: IPA (Instituto de Profesores Artigas), IFD (Institutos de Formación Docente), CERP (Centros Regionales de Profesores) e INET (Instituto Nacional de Enseñanza Técnica).

** Siguen 25 especialidades de carreras de profesorado.

Fuente: elaboración propia.

Para análisis de los datos derivados de las entrevistas recurrimos a la elaboración de un sistema de categorías interpretativas y explicativas (Miles y Huberman, 1984). Se utilizó el procedimiento propuesto por el método comparativo constante (Glaser y Strauss, 1967; Strauss, Corbin y Zimmerman, 2002). El plan de análisis consistió en partir de la información obtenida de las entrevistas y construir categorías (por ejemplo, modalidad de cursado) y subcategorías de análisis (percepción sobre el uso en el aula de formación inicial, infraestructura y calidad del dispositivo) en un proceso de abstracción creciente que incluyó tanto lo descriptivo como lo relacional.

\section{RESULTADOS}


Un primer hallazgo acerca de los resultados del estudio es que los docentes en formación tienen un acceso amplio a las tecnologías digitales. El 83\% ya recibió el laptop en el momento del estudio, un porcentaje similar accede en la actualidad a Internet desde el propio centro de formación y el $72 \%$ declara que tiene acceso a un teléfono celular tipo smartphone. El $50 \%$ de los estudiantes manifiesta que usa computadoras portátiles que le proporciona la propia institución formadora (como un segundo recurso, además de las entregadas a título personal por el Estado).

Según la valoración de los estudiantes consultados, los docentes formadores usan poco las tecnologías en sus prácticas pedagógicas y en general es reducido el número de docentes que le piden a los estudiantes de profesorado que utilicen el dispositivo en el aula. Al ser consultados sobre cuántos profesores les habían solicitado utilizar el ordenador en clase, al menos una vez en el último mes, los datos son muy elocuentes: el 46\% de los estudiantes encuestados nunca recibió instrucciones de incorporación de la tecnología en clase por parte de los profesores de las asignaturas del campo específico de la carrera de profesorado. En el mismo sentido, el $62 \%$ de los encuestados reveló que ningún docente de las ciencias de la educación (pedagogía, psicología, investigación educativa) le había pedido usar el ordenador portátil en el último mes. En los cursos de didáctica, espacio fundamental para la formación del futuro docente, ocurre lo mismo: el 57\% de los estudiantes nunca recibió orientaciones de usar el ordenador portátil en clase como apoyo al aprendizaje.

Con respecto a las cuestiones específicas referidas a la percepción sobre el impacto del uso de tecnologías en el aprendizaje, los estudiantes tienen una visión más positiva del uso de las tecnologías en general ( $85 \%$ ) que del uso específico de la computadora portátil (54\%) en las aulas de profesorado. Uno de cada cinco estudiantes $(23 \%)$ de la muestra son usuarios extremos de Internet.

El 55\% de los estudiantes consultados manifestó que al momento del relevamiento de datos, el ordenador portátil funcionaba correctamente. Esta evidencia coincide con otros estudios nacionales e internacionales (UNESCO, 2016a, 2017) que dan cuenta de las dificultades relacionadas con el mantenimiento de la infraestructura tecnológica de las políticas educativas que promueven el acceso universal y gratuito a las tecnologías.

\section{El uso de los ordenadores portátiles}

La Tabla 6, evidencia que los alumnos consultados tienen un uso medio del ordenador portátil cuando participan en las aulas del centro de formación inicial.

Tabla 6. Uso de ordenadores portátiles como dispositivo móvil en las aulas del centro de formación de profesorado.

\begin{tabular}{|l|l|l|l|l|l|l|}
\hline $\begin{array}{l}\text { Uso del dispositivo móvil } \\
\text { en.. }\end{array}$ & $\mathbf{X}$ & Mín. & Máx. & S.D. & $\begin{array}{c}\text { \% de uso } \\
\text { al menos } \\
\text { una vez a } \\
\text { la } \\
\text { semana }\end{array}$ & $\begin{array}{c}\text { Nunca usa } \\
\text { el } \\
\text { dispositivo }\end{array}$ \\
\hline $\begin{array}{l}\text { Aulas de pedagogía y ciencias } \\
\text { de la educación }\end{array}$ & 2.00 & 1 & 4 & 1.133 & $37.3 \%$ & $50.7 \%$ \\
\hline Aula de didáctica & 2.18 & 1 & 4 & 1.193 & $42.8 \%$ & $44.1 \%$ \\
\hline La práctica docente & 2.22 & 1 & 4 & 1.079 & $45 \%$ & $36 \%$ \\
\hline $\begin{array}{l}\text { Aulas de las asignaturas } \\
\text { específicas de la especialidad }\end{array}$ & 2.28 & 1 & 4 & 1.244 & $45.9 \%$ & $42.5 \%$ \\
\hline
\end{tabular}

Pregunta realizada a los estudiantes que usan el dispositivo en el centro. 1 (casi nunca), 2 (al menos una vez al mes), 3 (al menos una vez a la semana) y 4 (en todas o casi todas las clases).

Fuente: elaboración propia. 
En torno al $40 \%$ de los estudiantes relevados usa el recurso por lo menos una vez a la semana. Los datos indican además que un porcentaje importante del estudiantado nunca ha usado como apoyo el soporte digital proporcionado (oscilando entre un 50.7\% en el área de formación de la pedagogía y las ciencias de la educación a un $36 \%$ en la práctica docente. Entre las razones, los participantes consultados señalan las limitaciones técnicas del dispositivo personal entregado por las autoridades.

"Cuando vas a la realidad te encontrás con que las computadoras están descargadas, a los estudiantes se les rompió, que la llevaron a reparar y todavía no están reparadas, todos esos inconvenientes"” "el tema de los ordenadores portátiles hay que ir afinándolo a largo plazo. La idea de que estén (...) es genial, pero en realidad cuando las vas a usar no están las computadoras. Yo también tengo ordenador portátily se rompen, son lentas, se trancan, es como que bay que afinar esa parte".

Un segundo aspecto a resaltar es que, según la valoración del estudiantado, el uso es más frecuente en cuando éste está inmerso en su práctica pre profesional como docente $(\mathrm{M}=2.22$, $\mathrm{DS}=1.079)$ y cuando participa en el espacio formativo del área curricular especifica de su carrera de profesorado $(\mathrm{M}=2.28$, $\mathrm{DS}=1.244)$.

En este sentido, varios estudiantes destacaron la necesidad de lograr un mayor impacto en los estudiantes liceales (alumnos de la práctica docente) y promover un uso más intensivo de los recursos digitales:

"el efecto sería mayor sí más docentes los utilizáramos; por ejemplo, si de 10 docentes que tienen en la semana 9 utilizaran un blog, eso los motivaría para hacerlo con más asiduidad”.

Comentaron experiencias exitosas de introducción del ordenador portátil con un sentido pedagógico. Por ejemplo:

"Con los ordenadores he utilizado sensores. Actividades interactivas como por ejemplo las del programa KAJU, que les interesa bastante. Las uso cada tanto." "La primera experiencia me impresionó más..,(...), habiamos trabajado el tema de simetrías y fuimos con el grupo a un parque y luego a un museo, con los ordenadores. Ellos se dedicaron a sacar fotos, la parte más enriquecedora fue la bumana..., pero te diría que un $90 \%$ la uso casi todo el año en trigonometría".

"Para movimientos filmamos videos y utilizamos el programa Tracker para análisis o el Logger pro" "Por ejemplo bicimos programas en scratch, utilizamos Gimp". "Realizamos un stop motion utilizando Skratch en el ordenador portátil, y con el uso de tuxpaint realizaron composiciones. En grupos de 3er año de educación media estamos utilizando Tinkercad. Una aplicación web de dibujo 3 d”.

Las evidencias indican que el grado de autonomía del estudiante es un factor clave para participar en experiencias de aprendizaje móvil.

El tipo de uso y el espacio físico (dentro o fuera de la escuela) donde el estudiantado accede a los recursos utilizando como apoyo soportes informáticos portátiles fue una nueva dimensión considerada en la investigación.

Los datos coindicen con otros estudios internacionales que destacan un mayor uso fuera del centro (OECD, 2017) que en la propia institución formadora. La comparación de actividades seleccionadas se presenta en la Tabla 7. Si analizamos en detalle el tipo de actividad, cuando el estudiantado participa en las aulas de formación inicial usa el ordenador portátil fundamentalmente para la comunicación con otros estudiantes o profesores a través de redes sociales $(\mathrm{M}=2.52$, $\mathrm{DS}=1.200)$, navegar por la web $(\mathrm{M}=2.47, \mathrm{DS}=1.209)$ y crear presentaciones $(\mathrm{M}=2.25, \mathrm{DS}=1.050)$.

Tabla 7. Comparación del uso de ordenadores portátiles como dispositivo móvil dentro y fuera del centro educativo en actividades seleccionadas.

\begin{tabular}{|c|c|c|c|c|c|c|}
\hline \multicolumn{7}{|c|}{ EN EL CENTRO EDUCATIVO } \\
\hline $\begin{array}{c}\text { Utilizo el dispositivo } \\
\text { móvil para }\end{array}$ & $\mathrm{X}$ & Mín. & Máx. & S.D. & $\begin{array}{c}\% \text { de } \\
\text { uso al } \\
\text { menos } \\
\text { una vez }\end{array}$ & $\begin{array}{c}\text { Nunca usa } \\
\text { el } \\
\text { dispositivo }\end{array}$ \\
\hline
\end{tabular}

Educação em Revista | Belo Horizonte|v.36|e216356|2020 


\begin{tabular}{|c|c|c|c|c|c|c|}
\hline & & & & & $\begin{array}{c}\text { a la } \\
\text { semana }\end{array}$ & \\
\hline $\begin{array}{l}\text { Navegar por Internet para } \\
\text { el seguimientos de los } \\
\text { cursos }\end{array}$ & 2.47 & 1 & 4 & 1.209 & $42.7 \%$ & $33.2 \%$ \\
\hline $\begin{array}{l}\text { La comunicación con } \\
\text { otros estudiantes o el } \\
\text { profesor usando redes } \\
\text { sociales (Facebook, } \\
\text { WhatsApp, apps) }\end{array}$ & 2.52 & 1 & 4 & 1.200 & $57.4 \%$ & $32.9 \%$ \\
\hline $\begin{array}{l}\text { Usar software específico } \\
\text { de la especialidad de } \\
\text { profesorado }\end{array}$ & 1.92 & 1 & 4 & 1.111 & $31.6 \%$ & $52.5 \%$ \\
\hline $\begin{array}{l}\text { Crear presentaciones, } \\
\text { documentos, videos }\end{array}$ & 2.25 & 1 & 4 & 1.050 & $43 \%$ & $31.9 \%$ \\
\hline $\begin{array}{l}\text { Entrar a la } \\
\text { Plataforma } \\
\text { CREA2/EdModo/Moodle }\end{array}$ & 1.66 & 1 & 4 & 0.985 & $23.8 \%$ & $64.1 \%$ \\
\hline $\begin{array}{l}\text { Responder a tareas de } \\
\text { evaluación por parte del } \\
\text { profesor }\end{array}$ & 1.98 & 1 & 4 & 1.082 & $32.4 \%$ & $47.1 \%$ \\
\hline \multicolumn{7}{|c|}{ FUERA DEL CENTRO EDUCATIVO } \\
\hline $\begin{array}{l}\text { Navegar por Internet para } \\
\text { el seguimientos de los } \\
\text { cursos }\end{array}$ & 2.60 & 1 & 4 & 1.256 & $58.2 \%$ & $32.3 \%$ \\
\hline $\begin{array}{l}\text { La comunicación con } \\
\text { otros estudiantes o el } \\
\text { profesor usando redes } \\
\text { sociales (Facebook, } \\
\text { WhatsApp, apps) }\end{array}$ & 2.46 & 1 & 4 & 1.266 & $45 \%$ & $38.3 \%$ \\
\hline $\begin{array}{l}\text { Usar software específico } \\
\text { de la especialidad de } \\
\text { profesorado }\end{array}$ & 1.85 & 1 & 4 & 1.086 & $27.8 \%$ & $55.3 \%$ \\
\hline $\begin{array}{l}\text { Crear presentaciones, } \\
\text { documentos, videos }\end{array}$ & 2.15 & 1 & 4 & 1.133 & $38.5 \%$ & $40.9 \%$ \\
\hline $\begin{array}{l}\text { Entrar a la Plataforma } \\
\text { CREA } \\
\text { 2/EDdModo/Moodle }\end{array}$ & 2.18 & 1 & 4 & 1.168 & $40.9 \%$ & $41.6 \%$ \\
\hline $\begin{array}{l}\text { Responder a tareas de } \\
\text { evaluación por parte del } \\
\text { profesor }\end{array}$ & 2.24 & 1 & 4 & 0.069 & $42.9 \%$ & $39.9 \%$ \\
\hline
\end{tabular}

Pregunta realizada a los estudiantes que usan el dispositivo en el centro y fuera del centro: 1 (casi nunca), 2 (al menos una vez al mes), 3(al menos una vez a la semana) y 4 (en todas o casi todas las clases).

Fuente: elaboración propia.

Un dato llamativo es el escaso número de estudiantes que ingresan a las plataformas digitales a realizar tareas de estudio en el propio centro educativo (el acceso es mayor desde el hogar). Un tercio de los alumnos de profesorado (35.9\%) ingresan a las Plataformas de aprendizaje (Ed Modo, Plataforma Crea2) usando el ordenador portátil en el horario que concurre al centro. Este porcentaje asciende a $41.6 \%$ cuando el ingreso al sitio es desde el hogar. 
En concordancia con estudios previos (Rodríguez-Zidan, et al., 2017), los estudiantes usan poco las tecnologías para realizar actividades productivas o aplicativas, como lo es el caso de apoyarse en la incorporación de software específico vinculado con la especialidad de profesorado $(\mathrm{M}=1.92, \mathrm{DS}=1.111)$.

Responder a las tareas del profesor es una actividad que se lleva a cabo fundamentalmente desde el hogar $(\mathrm{M}=2.24$, DS $=0.004)$ en comparación con el uso en la propia clase en interacción con el profesor $(\mathrm{M}=1.98$, DS=1.082).

Este aspecto podría estar mostrando que muchas de las prácticas docentes realizadas por los docentes formadores remiten a propuestas pedagógicas con escasa inclusión de tecnología como recurso didáctico.

Con respecto a cómo valoran los estudiantes los efectos positivos y los desafíos de incorporar la tecnología en sus prácticas de aula como futuros profesores se analizaron los significados y las representaciones que emergen de las entrevistas.

Acerca de los efectos positivos del uso de herramientas digitales, y particularmente del ordenador portátil, los estudiantes refieren a los desafíos que implica abandonar los recursos tradicionales como el pizarrón y la tiza y adaptarse a la inclusión de recursos (programas, software, apps, uso de los ordenadores portátiles de los alumnos de educación media) con versatilidad y flexibilidad para la enseñanza mediada con tecnología. Señalaron que "el efecto sería mayor si más docentes las utilizáramos, la accesibilidady el trabajo en equipo serian aspectos favorecedores del uso de la tecnología, (...) para no sentirse tan solo como docente practicante".

\section{El modelo de Regresión}

Las estimaciones de modelos econométricos de elección discreta, conocidos como logit, permiten identificar las características determinantes de las decisiones de un grupo de individuos. Concretamente, las regresiones logit nos indican la dirección de las preferencias del grupo de estudiantes de carreras de pedagogía sobre características, usos y perfiles del aprendizaje basado en los laptops entregados por el Plan CEIBAL en Uruguay. A continuación, se presentan en la Tabla 8 dos estimaciones logit con la utilidad del ordenador portátil como la variable dependiente.

Tabla 8. Regresiones logit: Determinantes de la utilidad de los ordenadores portátiles.

\begin{tabular}{|l|c|c|c|c|c|c|c|c|}
\hline \multirow{2}{*}{$\begin{array}{l}\text { Variables en el } \\
\text { modelo }\end{array}$} & \multicolumn{4}{|c|}{ Regresión 1 } & \multicolumn{5}{c|}{ Regresión 2 } \\
\cline { 2 - 10 } & $\begin{array}{c}\text { Error } \\
\text { estándar }\end{array}$ & Sig. & $\mathbf{E x p ( B )}$ & B & $\begin{array}{c}\text { Error } \\
\text { estándar }\end{array}$ & Sig. & Exp(B) \\
\hline Género &,- 435 &, 375 &, 245 &, 647 & - & - & - & - \\
\hline Grupo de Edad &,- 531 &, 348 &, 127 &, 588 & - & - & - & - \\
\hline Modalidad &,- 658 &, 361 &, 069 &, 518 & $-0,768$ & 0,365 & 0,036 & 0,464 \\
\hline Especialidad &,- 327 &, 365 &, 371 &, 721 & - & - & - & - \\
\hline Nivel que cursa &, 371 &, 505 &, 462 & 1,450 & - & - & - & - \\
\hline $\begin{array}{l}\text { A partir del uso de } \\
\text { tecnologías } \\
\text { digitales } \\
\text { ¿considera que ha } \\
\text { cambiado su } \\
\text { forma de aprender } \\
\text { ? }\end{array}$ &, 818 &, 432 &, 058 & 2,267 & - & - & - & - \\
\hline $\begin{array}{l}\text { A partir del uso de } \\
\text { la computadora } \\
\text { portátil: } \\
\text { ¿considera que ha } \\
\text { cambiado }\end{array}$ & 2,572 &, 395 &, 000 & 13,091 & 2,447 & 0,37 & 0 & 11,549 \\
\hline
\end{tabular}




\begin{tabular}{|l|l|l|l|l|l|l|l|l|}
\hline $\begin{array}{l}\text { forma de aprender } \\
?\end{array}$ & & & & & & & & \\
\hline $\begin{array}{l}\text { ¿Has utilizado } \\
\text { aplicaciones } \\
\text { educativas (apps) } \\
\text { como apoyo al } \\
\text { aprendizaje? }\end{array}$ &, 795 &, 380 &, 036 & 2,214 & 0,889 & 0,353 & 0,012 & 2,432 \\
\hline $\begin{array}{l}\text { ¿Usa ordenadores } \\
\text { portátiles de la } \\
\text { Institución? }\end{array}$ & - & - & - & - & 1,22 & 0,347 & 0 & 3,386 \\
\hline Constante &,- 555 &, 667 &, 405 &, 574 & $-0,478$ & 0,35 & 0,172 & 0,62 \\
\hline
\end{tabular}

Fuente: elaboración propia.

El primer modelo estimado, contiene 8 variables, de las cuales sólo 2 resultaron ser significativas. En la segunda estimación, se eliminan las variables menos significativas hasta obtener una especificación de un modelo con aquellas variables que resultan ser más significativas en conjunto. Así, se conoce que los estudiantes de carreras de pedagogía en modalidad presencial tienen una percepción negativa de la utilidad del ordenador portátil con respecto a los estudiantes en semipresencial. Sin embargo, los estudiantes en general perciben que el uso de ordenador portátil les ha cambiado positivamente su forma de aprender. Además, los estudiantes que cuentan con ordenador portátil tienen mayor probabilidad de usar aplicaciones educativas como apoyo para el aprendizaje.

No se encontró evidencia significativa de que el género, la edad, la especialidad y el nivel cursado, sean características que influyan sobre la percepción de utilidad de los ordenadores portátiles.

Acerca de la primera categoría, los estudiantes resaltan la importancia de la coordinación entre los docentes en busca de criterios comunes y el trabajo en equipo entre los profesores, como factores que podrían potenciar el uso de estos dispositivos en el aula. Al respecto un estudiante de profesorado de Biología declaró:

"la accesibilidad y el trabajo en equipo serían aspectos favorecedores de su uso. Los cursos serían valiosos, pero sobre todo trabajar en equipos, para no sentirte tan solo como docente".

Con respecto a las limitaciones o barreras a superar para lograr un uso más eficiente de los dispositivos móviles los hallazgos refieren a aspectos vinculados con el aula de clases, las limitaciones técnicas del software del dispositivo y la infraestructura digital en los centros educativos. Con respecto al primer aspecto señalado, un estudiante reflexionó sobre las políticas TIC en formación de profesorado y los desafíos de la implementación pedagógica:

"Es como que es el "bum" el tema del Plan ceibal, repartir ordenadores portátiles y todo eso y a su vez fue bastante contradictorio con lo que iba pasado a nivel de la formación de nosotros como docentes, no fue bastante equilibrado. Yo lo que be aprendido y lo que aprendo porque necesito a veces para las clases en bastante autodidacta".

\section{DISCUSIÓN}

Las tecnologías digitales favorecen la irrupción en educación superior de nuevos modelos de aprendizajes (Becker et al., 2017) y representan una ventana abierta a nuevas alternativas y opciones de aprender (Schneckenberg, Ehlers y Adelsberger, 2011). Los dispositivos móviles, específicamente, devienen en escenarios educativos nuevos, tanto formales como informales (Hinostroza et al., 2016; UNESCO, 2016a, 2016b;), y contribuyen a la autorregulación del aprendizaje (Yot y Marcelo, 2017; Zheng, Li y Chen, 2016). Sin embargo, el uso que hacen de ellos 
docentes y estudiantes es limitado. Los resultados de este estudio nos presentan a un alumnado con un bajo nivel de uso del ordenador portátil.

El cuestionario midió la frecuencia de uso del ordenador portátil en cuatro tipos de uso del dispositivo móvil en el propio centro de formación del profesorado y otros siete tipos de uso tanto dentro como fuera del centro educativo. Nunca usaron el dispositivo proporcionado por la administración educativa el 36\% de los estudiantes en la práctica docente y el 50.7\% en los cursos de las asignaturas pedagógicas. La actividad realizada con mayor frecuencia, tanto dentro como fuera del centro educativo, es la navegación para el seguimiento de los cursos y la comunicación con los estudiantes o con el profesor a través de redes sociales (entre el $45 \%$ y el $58 \%$ utiliza el dispositivo para estos fines al menos una vez a la semana). No se hallaron diferencias significativas con respecto al uso de los dispositivos según las características consideradas en el modelo de regresión.

En concordancia con lo acontecido entre estudiantes universitarios al hacer uso de las tecnologías (Margaryan, Littlejohn y Vojt, 2011), el laptop sirve según lo manifestado por los encuestados para favorecer las relaciones sociales y ampliar el acceso al contenido conceptual. Rossing et al. (2012) apuntaban al aumento del acceso a la información como consecuencia del uso de dispositivos móviles en el aula. Tanto dentro como fuera del aula, ésta es una de las dos actividades que más se practica semanalmente por los docentes en formación.

Esto se explica, en parte, porque el alumnado dispone de un conocimiento limitado sobre el uso que de la tecnología puede hacer para el aprendizaje y esto es congruente con otras investigaciones (Lai y Gu, 2011). Desconocen la utilidad formativa que las tecnologías tienen, no comprenden cómo emplearlas eficazmente para apoyar su propio aprendizaje (Littlejohn, Margaryan y Vojt, 2010).

Nuestros hallazgos coinciden con el estudio de Gong y Wallace (2012) el cual reveló que los dispositivos móviles son más usados para entretenimiento que para fines educativos. Es por ello que, para que los estudiantes las adopten para favorecer su propio aprendizaje, necesitan orientación sobre cómo hacerlo (Deepwell y Malik, 2008). Y esto pasa por incorporarlas a la práctica formativa.

Ahora bien, mientras que algunos formadores lo han aceptado y están buscando la forma de integrarlos, otros han puesto la idea a invernar o la han rechazado de plano (Foulger et al., 2013). Así, hemos hallado que los docentes de las áreas de especialización lo emplean más que los docentes responsables de la formación pedagógica de los futuros docentes.

\section{CONCLUSIONES}

Los principales hallazgos de este estudio revelan que los estudiantes de profesorado declaran una baja frecuencia de uso del ordenador portátil distribuido gratuitamente por la política educativa siendo mayor el uso en las asignaturas de formación específica de la especialidad frente a las asignaturas pedagógicas. Estos resultados concuerdan con lo propuesto por otras investigaciones que destacan el escaso uso pedagógico que hacen los profesores y estudiantes de profesorado de la tecnología ubicua. (Uerz, Volman y Kral, 2018).

El futuro diseño de escenarios disruptivos para el aprendizaje de la profesión docente necesita de nuevos estudios aplicados que aporten conocimiento específico acerca de cuál es el grado de avance, el acceso, uso efectivo y penetración de la tecnología en las aulas y en las prácticas educativas en la marco de las políticas de acceso universal a la tecnología como los modelos de distribución gratuita de una computadora por alumno.

Finalmente, debe señalarse que una de las limitaciones de este estudio es que sólo se indaga la perspectiva de los estudiantes. A efectos de enriquecer el análisis es necesario complementar los hallazgos con la visión de otros actores, como los docentes responsables de implementar las innovaciones TIC a nivel de cada centro, los directores y los profesores formadores de los diferentes campos curriculares considerados. 


\section{REFERÊNCIAS}

Aslan, A. y Zhu, C. (2015). Pre-Service Teachers' Perceptions of ICT Integration in Teacher Education in Turkey. The Turkish Online Journal of Educational Technology, 14(3), 97-110.

Aubusson, P., Schuck, S., y Burden, K. (2009). Mobile learning for teacher professional learning: benefits, obstacles and issues. Research in Learning Technology, 17(3). DOI: https://doi.org/10.3402/rlt.v17i3.10879

Avidov-Ungar, O. y Iluz, I. E. (2014). Levels of ICT integration among teacher educators in a teacher education academic college. Interdisciplinary Journal of E-Learning and Learning Objects, 10, 195-216.

Bakir, N. (2016). Technology and Teacher Education: A Brief Glimpse of the Research and Practice that Have Shaped the Field. TechTrends, 60(1), 21-29. DOI: 10.1007/s11528- 0150013-4

Baran, E. (2014). A Review of Research on Mobile Learning in Teacher Education. Journal of Educational Technology \& Society, 17(4), 17-32, http://www.jstor.org/stable/jeductechsoci.17.4.17 [Consulta: oct. 2018].

Becker, S.A., Cummins, M., Davis, A., Freeman, A., Glesinger Hall, C. y Ananthanarayanan, V. (2017). NMC Horizon Report: 2017 Higher Education Edition. Austin, Texas: The New Media Consortium, https://www.learntechlib.org/p/174879/ [Consulta: oct. 2018].

Brecko, B. N., Kampylis, P. y Punie, Y. (2014). Mainstreaming ICT-enabled Innovation in Education and Training in Europe: Policy actions for sustainability, scalability and impact at system level. JRC Working Papers JRC83502, Joint Research Centre (Seville site), https://ideas.repec.org/p/ipt/iptwpa/jrc83502.html [Consulta: oct. 2018].

Cabrera Borges, C. A., Rodriguez Zidán, C. E. y Zorrilla Salgador, J. P. (2018). Integración de dispositivos móviles en la formación inicial y en las prácticas educativas de los estudiantes de profesorado de Uruguay. Revista Latinoamericana de Educación Comparada, 9(14), pp 123-141.

Creswell, J. W., y Plano Clark, V. L. (2007). Designing and conducting Mixed Methods research. Thousand Oaks, CA: Sage. DOI:10.1177/1558689807306132

Chaia, A., Child, F., Dorn, E., Frank, M., Krawitz, M. y Mourshed, M. (2017). What Drives Student Performance in Latin America?, McKinsey Report, https://www.mckinsey.com/industries/social-sector/our-insights/what-drives-studentperformance-in-latin-america [Consulta: oct. 2018].

Deepwell, F., y Malik, S. (2008). On campus, but out of class: an investigation into students' experiences of learning technologies in their self-directed study. Research in Learning Technology, 16(1). DOI: https://doi.org/10.3402/rlt.v16i1.10881

Donovan, L., Green, T. y Hansen, L. E. (2011). One-to-One Laptop Teacher Education, Journal of Research on Technology in Education, 44(2), 121139, DOI: $10.1080 / 15391523.2011 .10782582$

Dyson, L. E., Litchfield, A., Lawrence, E., Raban, R., y Leijdekkers, P. (2009). Advancing the m-learning research agenda for active, experiential learning: Four case studies. Australasian Journal of Educational Technology, 25(2). DOI: https://doi.org/10.14742/ajet.1153

Ferguson, R. y Buckingham, S. (2012). Social learning analytics: five approaches. In: 2nd International Conference on Learning Analytics \& Knowledge, 29 Apr - 02 May 2012, Vancouver, British Columbia, Canada, 23-33. DOI: https://doi.org/10.1145/2330601.2330616

Fernández, C., Cebreiro, B., y Fernández, J. C. (Dirs.) (2014). State of the art of ICT integration in European Schools educational Systems. Equipment-Uses-Teachers Competences. Research Report, http://icteacher.eu/fileadmin/images/downloads/FINAL RESEARCH REPORT.pdf [Consulta: oct. 2018].

Flick, U. (2015). El diseño de la investigación cualitativa. Madrid: Alianza Editorial. 
Foulger, T.S., Burke, D., Williams, M.K.; Waker, M.L.; Hansen, R. y Slykhuis, D.A. (2013). Innovators in Teacher Education, Journal of Digital Learning in Teacher Education, 30:1, 21-29, DOI: https://doi.org/10.1080/21532974.2013.10784722

Fullan, M., y Langworthy, M. (2013). Towards a New End: New Pedagogies for Deep Learning. Seattle, Washington, USA, http://www.newpedagogies.org/Pages/assets/newpedagogies-for-deep-learning---an-invitation-to-partner-2013-19-06.pdf [Consulta: oct. 2018].

Galperin, H. (2017). Digital Society: Gaps and Challenges for Digital Inclusion in Latin America and the Caribbean. UNESCO Policy Papers. Recuperado el 3 de agosto de 2018 de http://unesdoc.unesco.org/images/0026/002628/262860e.pdf

Glaser, B. y Strauss, A. (1967). El muestreo teórico. En B., Glaser y A., Strauss. The Discovery, of grounded theory: strategies for qualitative research. (pp. 45-77). New York: Aldine Publishing Company.

Gong, Z. y Wallace, J. D. (2012). Gong, Z., \& Wallace, J. (2012). A Comparative analysis of iPad and other m-learning technologies: Exploring students' view of adoption, potentials, and challenges. Journal of Literacy and Technology, 13(1), 2-29.

Gülbahar, Y. (2008). ICT usage in higher education: a case study on preservice teachers and instructors. Turkish Online Journal of Educational Technology, 7(1), 32-37.

Gutiérrez, A., Palacios, A., y Torrego, L. (2010). La formación de los futuros maestros y la integración de las TIC en la educación: anatomía de un desencuentro. Revista de Educación, 353, $267-$ 293.

Hammond, M. (2014). Introducing ICT in schools in England: Rationale and consequences. British Journal of Educational Technology, 45(2), 191-201.DOI: 10.1111/bjet.12033

Herro, D., Kiger, D. y Owens, C. (2013). Mobile Technology. Journal of Digital Learning in Teacher Education, 30(1), 30-40. DOI: 10.1080/21532974.2013.10784723

Hinostroza, J. E., Ibieta, A., Claro, M., y Labbé, C. (2016). Characterisation of teachers' use of computers and Internet inside and outside the classroom: The need to focus on the quality. Education and Information Technologies 21(6), 1595-1610. DOI: https://doi.org/10.1007/s10639-0159404-6.

Hinostroza, J. E., Labbé, Ch., Brun, M., y Matamala, C. (2011). Teaching and learning activities in Chilean classrooms: Is ICT making a difference?, Computers \& Education, 57(1) 135867. DOI: https://doi.org/10.1016/i.compedu.2011.01.019.

Hughes, J. E. (2013). Descriptive indicators of future teachers' technology integration in the PK-12 classroom: trends from a laptop-infused teacher education program. Journal of Educational Computing Research, 48(4), 491-516. DOI: https://doi.org/10.2190/EC.48.4.e.

Hughes, J. E., Liu, S. y Lim, M. (2016). Technological modeling: Faculty use of technologies in preservice teacher education from 2004 to 2012. Contemporary Issues in Technology and Teacher Education, 16(2), 184-207.

Jara, I. (2015). Revisión comparativa de iniciativas nacionales de aprendizaje móvil en América Latina. El caso del Plan Ceibal de Uruguay. UNESCO, http://unesdoc.unesco.org/images/0024/002439/243976s.pdf [Consulta: oct. 2018].

Jisu, H., DeLorme, D.E. y Reid, L.N. (2006). Perceived Third-Person Effects and Consumer Attitudes on Prevetting and Banning DTC Advertising. Journal of Consumer Affairs, 40(1), 90-116. DOI:10.1111/j.1745-6606.2006.00047.x

Kalonde, G. y Mousa, R. (2016). Technology Familiarization to Preservice Teachers: Factors that Influence Teacher Educators' Technology Decisions. Journal of Educational Technology Systems, 45(2), 236-255. DOI: https://doi.org/10.1177/0047239515616965.

Kay, R. H. y Knaack, L. (2005). A Case for Ubiquitous, Integrated Computing in Teacher Education. Technology, Pedagogy and Education, 14(3), 391-412. DOI: 10.1080/14759390500200213.

Kopcha, T. J., Rieber, L. P., y Walker, B. B. (2016). Understanding university faculty perceptions about innovation in teaching and technology. British Journal of Educational Technology, 47(5), 945- 957. DOI: https://doi.org/10.1111/bjet.12361 
Lai, C. y Gu, M. (2011). Self-regulated out-of-class language learning with technology, Computer Assisted Language Learning, 24:4, 317-335, DOI: https://doi.org/10.1080/09588221.2011.568417

Lai, C., Wang, Q. y Lei, J. (2012). What factors predict undergraduate students' use of technology for learning? A case from Hong Kong. Computers \& Education, 59(2), 569-579. DOI: https://doi.org/10.1016/i.compedu.2012.03.006.

Littlejohn, A., Margaryan, A., y Vojt, G. (2010). Exploring Students' use of ICT and Expectations of Learning Methods. Electronic Journal of e-Learning, 8(1), 13-20.

Liu, O. L., Wilson, M. y Paek, I. (2008). A Multidimensional Rasch Analysis of Gender Differences in PISA Mathematics. Journal of Applied Measurement, 9 (1), 18-35.

Margaryan, A., Littlejohn, A., y Vojt, G. (2011). Are digital natives a myth or reality? University students' use of digital technologies. Computers \& Education, 56(2), 429-440. DOI: https://doi.org/10.1016/i.compedu.2010.09.004.

Mcloughlin, C. (2013). Teacher professional learning in digital age environments. En Back to the Future: Legacies, Continuities and Changes in Educational Policy, Practice and Research, editado por Maria Assunção Flores, Ana Amélia Carvalho, Fernando Ilídio Ferreira, y Maria Teresa Vilaça, 189-206. Rotterdam: SensePublishers. DOI: https://doi.org/10.1007/978-94-6209-240-2 11.

Messina, L., y Tabone, S. (2013). Technology proficiency, TPACK and beliefs about technology: A survey with primary school student teachers. REM-Research on Education and Media, 5(1), 11-29, http://ojs.pensamultimedia.it/index.php/rem en/article/view/1413 [Consulta: oct. 2018].

Miles, M. y Huberman, A.M. (1984). Qualitative data analysis. A source book of new methods, Beverly Hills, Sage.

Newhouse, C. P., Williams, P. J. y Pearson, J. (2006). Supporting mobile education for preservice teachers. Australasian Journal of Educational Technology, 22(3), 289-311.

OECD. (2017). PISA 2015 Results (Volume III): Students' well-being. Paris: Organisation for Economic Co-operation and Development (OECD).

Peng, C. Y., y So, T. S. H. (2002). Modeling Strategies In Logistic Regression With SAS, SPSS, Systat, BMDP, Minitab, And STATA. Journal of Modern Applied Statistical Methods, 1(1), $147-$ 156. DOI: https://doi.org/10.22237/imasm/1020255720.

Pittaluga, L. y Rivoir, A. (2012). One Laptop per Child and Bridging the Digital Divide: The Case of Plan CEIBAL in Uruguay. Information Technologies \& International Development, 8(4), 145159.

Ramírez-Montoya, M. S. y García-Peñalvo, F. J. (2017). La integración efectiva del dispositivo móvil en la educación y en el aprendizaje. RIED. Revista Iberoamericana de Educación a Distancia, 20(2), 29-47. DOI: https://doi.org/10.5944/ried.20.2.18884.

Rodríguez-Zidan, E; Marcelo, C; Bernasconi, G; Yot, C; Teliz, F; Umpiérrez, S. (2017). Educadores en la era digital: aprender a enseñar con tecnologias en la formación inicial de profesores de educación media en Uruguay. Montevideo, Uruguay. Recuperado de: https://digital.fundacionceibal.edu.uy/jspui/handle/123456789/222

Rossing, J. P., Miller, W. M., Cecil, A. K., y Stamper, S. E. (2012). iLearning: The future of higher education? Student perceptions on learning with mobile tablets. Journal of the Scholarship of Teaching and Learning, 12(2), 1-26, https://scholarworks.iu.edu/journals/index.php/josotl/article/view/2023 [Consulta: oct. 2018].

Sad, S. N. y Gokkta s, O. (2014). Preservice teachers' perceptions about using mobile phones and laptops in education as mobile learning tools. British Journal of Educational Technology, 45(4), 606618. DOI: $10.1111 /$ bjet.12064

Schneckenberg, D., Ehlers, U., y Adelsberger, H. (2011). Web 2.0 and competenceoriented design of learning. Potentials and implications for higher education. British Journal of Educational Technology, 42(5), 747-762. DOI: https://doi.org/10.1111/j.1467-8535.2010.01092.x 
Sipilä, K. (2010). The impact of laptop provision on teacher attitudes towards ICT. Technology, Pedagogy and Education, 19(1), 3-16. DOI: 10.1080/14759390903579257.

Strauss, A.; Corbin, J. y Zimmerman, E. (2002). Bases de la investigación cualitativa: técnicas y procedimientos para desarrollar la teoría fundamentada. Medellín: Universidad de Antioquia.

Sung, Y. T. Chang, K. E. y Liu, T. C. (2016). The effects of integrating mobile devices with teaching and learning on students' learning performance: A meta-analysis and research synthesis. Computers \& Education, 94, 252-275. DOI: https://doi.org/10.1016/j.compedu.2015.11.008.

Sunkel, G.; Trucco, D. y Espejo, A. (2014). La integración de las tecnologías digitales en las escuelas de América Latina y el Caribe. Una mirada multidimensional. Santiago de Chile: CEPAL.

Uerz, D. Volman,M. y Kral, M. (2018). Teacher educators' competences in fostering student teachers' proficiency in teaching and learning with technology: An overview of relevant research literature. Teaching and Teacher Education, 70, 12-23. DOI: https://doi.org/10.1016/j.tate.2017.11.005.

UNESCO (2013). El futuro del aprendizaje móvil. Implicaciones para la planificación y formulación de políticas. París, Francia: Organización de las Naciones Unidas para la Educación, la Ciencia y la Cultura.

UNESCO (2016a). Revisión comparativa de iniciativas nacionales de aprendizaje móvil en América Latina. El caso del Plan Ceibal de Uruguay. Buenos aires. UNESCO

UNESCO (2016b). Revisión comparativa de iniciativas nacionales de aprendizaje móvil en América Latina Organización de las Naciones Unidas para la Educación, la Ciencia y la Cultura. Los casos de Colombia, Costa Rica, Perú y Uruguay. IIPE Buenos Aires. UNESCO

UNESCO (2017). E2030: Education and skills for the 21st century. Regional Meeting of Ministers of Education of Latin America and the Caribbean. Buenos Aires, Argentina, January $24-25$, UNESCO.

Witecki, G., y Nonnecke, B. (2015). Engagement in digital lecture halls: A study of student course engagement and mobile device use during lecture. Journal of Information Technology Education: Research, 14, 73-90. Retrieved from http://www.jite.org/documents/Vol14/JITEv14ResearchP073-090Witecki0720.pdf

Yot, C. y Marcelo, C. (2017). University students' self-regulated learning using digital technologies. International Journal of Educational Technology in Higher Education, 14(38), 1-18. DOI: https://doi.org/10.1186/s41239-017-0076-8

Yusop, F. D. (2015). A dataset of factors that influence preservice teachers' intentions to use Web 2.0 technologies in future teaching practices. British Journal of Educational Technology, 46(5), 1075-1080. doi:10.1111/bjet.12330

Zheng, L., Li, X. y Chen, F. (2016). Effects of a mobile self-regulated learning approach on students' learning achievements and self-regulated learning skills. Journal Innovations in Education and Teaching International, 40(7), DOI: 10.1080/14703297.2016.1259080

Submetido: $10 / 11 / 2018$

Aprovado: 05/09/2019 